\title{
Vitamin A deficiency in chickens
}

\author{
By MARGARET B. AYDELOTTE \\ Physiological Laboratory, University of Cambridge \\ (Received 3 August 1962-Revised 12 December 1962)
}

The importance of vitamin A for the normal development and maintenance of skeletal, nervous and epithelial tissues has long been recognized, and the general signs of deficiency of this vitamin in chickens have been described by Seifried (I930) and by Beach (1923). One of the most important changes caused by vitamin A deficiency, often leading to serious infection, is the replacement of many secretory epithelia by non-secretory, keratinizing membranes.

In the experiments reported here, the effects of vitamin A deficiency on the corneal, conjunctival, oesophageal and tracheal epithelia of the young chick have been studied. In many animals these epithelia show metaplastic changes in vitamin A deficiency. Xerophthalmia (i.e. drying of the eyes, with keratinization of the corneal and conjunctival epithelia) was one of the first signs recognized as characteristic of vitamin $A$ deficiency, and in many species, e.g. the rat, it is often one of the earliest and most obvious changes to appear. In vitamin A-deficient chickens, gross changes can be seen in the eyes (Beach, 1923), but these lesions are not as extensive or as obvious as they sometimes are in rats and many other mammals.

Metaplastic changes also occur in the mucous glands of the chick oesophagus in vitamin A deficiency (Beach, I923; Seifried, 1930); in the trachea the epithelium, which is normally ciliated and secretory, gradually becomes stratified and keratinized (Seifried, 1930).

It was thought desirable to study the effects of vitamin A deficiency on these epithelia in more detail, however, for comparison with changes in the same tissues grown as organ cultures and subjected to varying concentrations of vitamin $A$. The results of these tissue-culture experiments, and a comparison between the epithelial changes observed in vitro and in vitamin A-deficient chicks, are presented in another publication (Aydelotte, 1963).

\section{EXPERIMENTAL}

From the time of hatching, a group of twenty-four chicks was kept on a diet low in vitamin A, and a control group was given the same basic diet, but with a higher supplementary dose of vitamin A. The basic diet was the same as that used by Elvehjem \& Neu (1932) for the production of avitaminosis A in chicks. The diet was supplemented with provitamin $D_{3}$ (O.I $\mathrm{mg} 7$-dehydrocholesterol $/ \mathrm{kg}$ dry food) for the first $3 \frac{1}{2}$ weeks, and with vitamin $D_{2}$ (4000 i.u. ergocalciferol $/ \mathrm{kg}$ dry food) for the remaining 9 weeks of the experiment. Although vitamin $D_{3}$ (cholecalciferol) is much more effectively utilized by the chick than is vitamin $D_{2}$, nevertheless the diet provided 
a daily intake of vitamin $D_{2}$ considerably above the minimal protective dose $\left(8_{5}\right.$ i.u./ chick daily) recommended by Remp \& Marshall (1938). Further, the chicks were frequently placed in direct sunlight for several hours at a time. As no signs of rickets were observed, it was assumed that the birds' need for vitamin $\mathrm{D}$ was being adequately met. For the control group, I0000 i.u. vitamin A (Crooke's veterinary product) were added $/ \mathrm{kg}$ dry food. The chicks on the low-vitamin A diet received $75^{\circ}$ i.u. $/ \mathrm{kg}$ food for the first 3 weeks, which was slightly less than the minimum dose of I 100 i.u. vitamin $\mathrm{A} / \mathrm{kg}$ food that has given satisfactory growth in chicks for the first 8 weeks (Castano, Boucher \& Callenbach, 195I). After the withdrawal of vitamin A from the diet of the deficient group, some chicks that had become noticeably deficient were given intramuscular injections of 1000 i.u. vitamin A to prevent early death.

Material for histological examination was taken when a chick showed characteristic signs of deficiency. Post-mortem material from chicks that had died from vitamin A deficiency was also examined, and tissue for comparison was taken from control chicks of the same age. The tissues were dissected from the chick, washed briefly in Tyrode's solution, fixed in Zenker-formol or Susa's fixative and embedded in paraffin wax. Sections were cut $5-7 \mu$ thick and stained with Mayer's acid haemalum and alcian blue or with periodic acid Schiff (PAS) and haemalum.

\section{RESULTS}

Characteristic signs of deficiency were first seen in the deficient group 2 weeks after the withdrawal of vitamin A from the diet: the chicks ceased growing, becoming ataxic and drowsy, and their feathers appeared ruffled. The chicks of the control group remained healthy and continued to gain weight during the $12 \frac{1}{2}$ weeks of the experiment.

The eyelids of the vitamin A-deficient chicks were drier and open less wide than usual; although these chicks did not show true xerophthalmia, histological changes were always found in the corneal and conjunctival epithelia. The cornea in the control chicks was smooth, and the superficial nucleated cells showed no sign of desquamating, but the superficial cells of the deficient chicks were vacuolated and desquamating, making the cornea slightly rough.

The conjunctival epithelium frequently became keratinized in the deficient chicks. In the normal eye, there was a fairly abrupt transition from the stratified, squamous epithelium of the cornea to the folded, secretory conjunctiva ( $\mathrm{Pl}$. $\mathrm{x} a$ ). This cornealconjunctival junction was sensitive to deficiency of vitamin A. In less affected chicks, the conjunctival epithelium immediately round the cornea lost its secretory cells and became thicker, but with more severe deficiency a thick layer of keratin developed over the conjunctiva and protruded above the surface of the cornea (Pl. $\mathrm{I} b, c)$. The more severe the deficiency, the greater was the keratinized area of the bulbar conjunctiva.

The oesophageal epithelium of the control chicks was thick, stratified, squamous and non-keratinized. The simple alveolar mucous glands in the lamina propria opened to the surface through ducts lined by cuboidal mucous cells (Pl. 2a). 
In the deficient chicks, the surface of the oesophageal mucosa, when seen with the naked eye, appeared pale and dry with small white patches, in contrast to the normal mucosa, which was pink and moist with mucus. Histologically, there was little change in the surface of the oesophageal epithelium in vitamin A deficiency, but marked changes could be seen in the mucous glands: in the early stages of deficiency, the stratified, squamous epithelium gradually extended down the ducts towards the glands, and the lining secretory cells of the ducts began to atrophy. As the deficiency progressed, the squamous metaplastic changes spread into the secretory acini (P1. $2 b)$; eventually the glands were completely replaced by stratified, keratinizing epithelium (P1. 2c). The ducts and acini often became blocked and filled with keratinizing cells, debris and mucus, forming white cysts visible to the naked eye.

The tracheal epithelium of the control chicks was pseudostratified, with groups of tall, columnar, ciliated cells alternating with intra-epithelial mucous glands (Pl. $3 a$ ). The small basal cells showed few mitoses.

Changes in the tracheal epithelium resulting from vitamin A deficiency could be detected in all specimens examined histologically, although the degree of damage varied from one chick to another and in different regions of the same trachea. The chicks could be divided roughly into three groups, according to the extent of the change:

(a) Those least affected showed in some regions an increase in the size of the mucous glands and individual mucous cells, together with a decrease in the ciliated surface area $\left(\mathrm{P} 1.3^{b}\right)$. Other parts of the epithelium still appeared fairly normal.

(b) In more severe deficiency, patches of the tracheal epithelium became stratified, as a result of increased basal cell division. In these patches, the original ciliated and mucous cells were separated from the basement membrane by two to three layers of undifferentiated cells; often these superficial cells showed an increase in the proportion of mucous to ciliated cells. Other regions of the epithelium in these chicks were still pseudostratified, but showed increased mucification, as in group (a).

(c) In more advanced deficiency, almost the whole tracheal lining became stratified, and mitoses were common amongst the basal cells (Pl. $3 c$ ). Small patches of ciliated cells and large mucous cells could still be found on the surface of the stratified epithelium, but elsewhere the normal epithelial cells were either degenerating and much flattened or had already been shed, leaving a stratified, squamous epithelium. Proliferation of the tracheal basal cells began in discrete foci and gradually extended through the whole epithelium.

\section{DISCUSSION}

Many of the changes observed in this experiment are like those previously found in vitamin A-deficient chicks. Although the corneal epithelium is normally stratified and squamous, it did not keratinize in these deficient chicks, whereas the conjunctiva was affected earlier and to a much greater degree. Mori $(1922 a, b)$ also found that in vitamin A deficiency loss of secretory cells and cornification occurred earliest in the conjunctiva bulbi of rats and spread from there to other parts of the conjunctiva and cornea. He concluded that the main effect of deficiency was lack of secretion by the 
para-ocular glands and that xerophthalmia was only a secondary condition resulting from this lack. It seems more likely, however, that vitamin A deficiency stimulates division of the basal cells of the conjunctival epithelium, causing thickening and keratinization, besides affecting the mucous cells; both these effects of vitamin A deficiency seem to be important in causing xerophthalmia.

In the oesophageal epithelium, the most severe changes during vitamin A deficiency were in the secretory portions, the mucous glands and their ducts. The changes described here are essentially the same as those reported by Seifried (1930), and they show the epithelial cells that normally become secretory to be capable of an alternative differentiation, with the production of keratin instead of mucus.

In severely deficient chicks, the changes in the tracheal epithelium were similar to those in the conjunctiva and oesophageal glands (i.e. replacement of secretory cells by a stratified, keratinizing epithelium), an observation in agreement with that of Seifried (1930). But during the early stages of deficiency there was an apparent increase in both the synthesis of mucus and the proportion of mucous cells to ciliated cells in the tracheal epithelium. As far as I am aware, this change has not previously been described as characteristic of partial vitamin A deficiency. Jungherr (r943) studied the histological changes in the chick in partial vitamin A deficiency, and noticed dilatation of the intra-epithelial mucous glands of the respiratory epithelium of the nose and increased mucification of some salivary glands, but he reported these changes as evidence of infection in the birds. Although increased mucification does undoubtedly occur in respiratory infections, it is possible that it can also be a direct response to vitamin A deficiency, unrelated to infection, and it may help to compensate for the degeneration of secretory cells from other regions of the epithelium.

These results suggest that vitamin A deficiency affects the tracheal epithelium in two stages: partial deficiency causes increased synthesis and secretion of mucus, and further depletion of vitamin A causes hyperplasia of the basal cells and a consequent development of stratified epithelium. As the original ciliated and mucous cells become separated from supplies of nutrients by the rapidly dividing basal cells, they gradually degenerate, leaving a stratified, squamous epithelium, which sometimes keratinizes.

It was previously believed that deficiency of vitamin $A$ inhibited secretion from most, if not all, mucous membranes, but the results of this experiment suggest that there is an optimal concentration of vitamin A for the synthesis of mucus by the tracheal epithelium, lower than the level to which this epithelium is normally exposed in the body. Similarly, secretion of mucus by other epithelia may be maximal at a particular concentration of vitamin A, but in both the conjunctival and oesophageal epithelia this level seems to be approximately the same as, or higher than, the normal concentration in the body. This theory is further supported by the results of experiments in which organ cultures of these tissues were grown in media containing different concentrations of vitamin A (Aydelotte, 1963). 


\section{SUMMARY}

I. The effects of vitamin A deficiency on chick corneal, conjunctival, oesophageal and tracheal epithelia are reported.

2. The corneal epithelium was only slightly affected by vitamin A deficiency, but the adjacent conjunctival epithelium lost its secretory cells and became keratinized.

3. The oesophageal mucous glands and their ducts were progressively replaced from the surface by stratified, keratinizing epithelium.

4. In the early stages of vitamin A deficiency, an increase in the size of the mucous cells, and in the proportion of mucous to ciliated cells, was observed in the tracheal epithelium. With more severe deficiency, these large secretory cells were shed from the surface as the epithelium became stratified and keratinized.

5. In the discussion it is suggested (a) that the optimal level of vitamin A for mucus secretion by the tracheal epithelium is lower than that to which it is normally exposed in the body, and $(b)$ that each mucus-secreting epithelium achieves maximal synthesis and secretion at a particular concentration of vitamin $A$.

The author thanks Dr E. N. Willmer, FRS, for his kind guidance and encouragement in this work. Financial assistance from the Medical Research Council (scholarship for training in research methods) is gratefully acknowledged.

\section{REFERENCES}

Aydelotte, M. B. (1963). F. Embryol. exp. Morph. II, 279.

Beach, J. R. (1923). Science, 58, 542.

Castano, F. F., Boucher, R. V. \& Callenbach, E. W. (I95 I). F. Nutr. 45, I3 I.

Elvehjem, C. A. \& Neu, V. F. (1932). F. biol. Chem. 97, 71.

Jungherr, E. (1943). Bull. Storrs agric. Exp. Sta. no. 250.

Mori, S. (1922a). F. Amer. med. Ass. 79, 197.

Mori, S. (1922b). Fohns Hopk. Hosp. Bull. 33, 357.

Remp, D. G. \& Marshall, I. H. (1938). F. Nutr. I5, 525.

Seifried, O. (1930). F. $\exp$. Med. 52, 519.

\section{EXPLANATION OF PLATES}

Photomicrographs of sections stained with haemalum and alcian blue: mucus appears dark.

\section{Plate I}

(a) Cornea and conjunctiva of a control chick. The arrow shows the point of transition from the cornea (on the left) to the conjunctiva (on the right).

(b) Cornea and conjunctiva of a vitamin A-deficient chick. The cornea is on the left and the conjunctiva on the right as in (a). The conjunctival epithelium is much thicker than in the control chick, and the surface is heavily keratinized.

(c) Central part of $(b)$ shown at higher magnification. Note the thick layer of keratin $(K)$ on the surface of the conjunctival epithelium.

\section{Plate 2}

(a) An oesophageal mucous gland and part of its duct from a control chick.

(b) An oesophageal mucous gland from a vitamin A-deficient chick. The mucous cells of the duct and acini have been partly replaced by a keratinizing epithelium. The dark patches in the peripheral parts of the gland are remaining secretory portions.

(c) An oesophageal gland at a later stage of vitamin A deficiency than that shown in (b). The mucous gland has become a keratinized cyst, filled with cell debris, keratin and mucus. 


\section{Plate 3}

(a) The tracheal epithelium of a control chick. The surface is ciliated, and the mucous cells (appearing dark) lie in hollows forming intra-epithelial glands.

(b) The tracheal epithelium of a partially vitamin A-deficient chick. Note the increase in size of the mucous glands as compared with those shown in $(a)$. Two small patches of ciliated cells $(C)$ remain between the glands.

(c) The tracheal epithelium at a later stage of vitamin A deficiency than that shown in (b). The basal cells are dividing rapidly (arrows point to mitotic figures) and the epithelium is stratified. A few flattened mucous cells $(M)$ remain on the surface. 


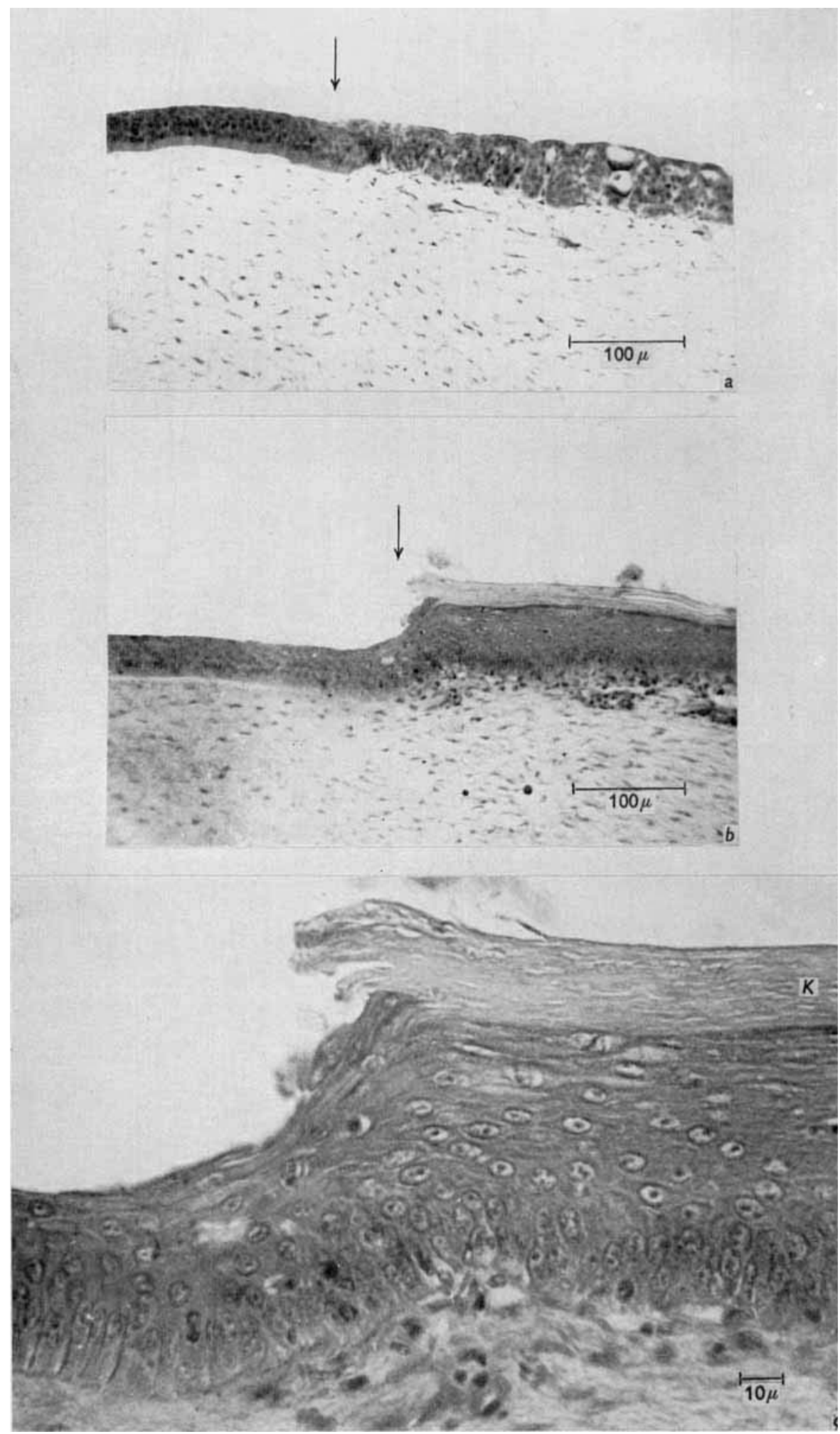


British Journal of Nutrition, Vol. I 7, No. 2

Plate 2
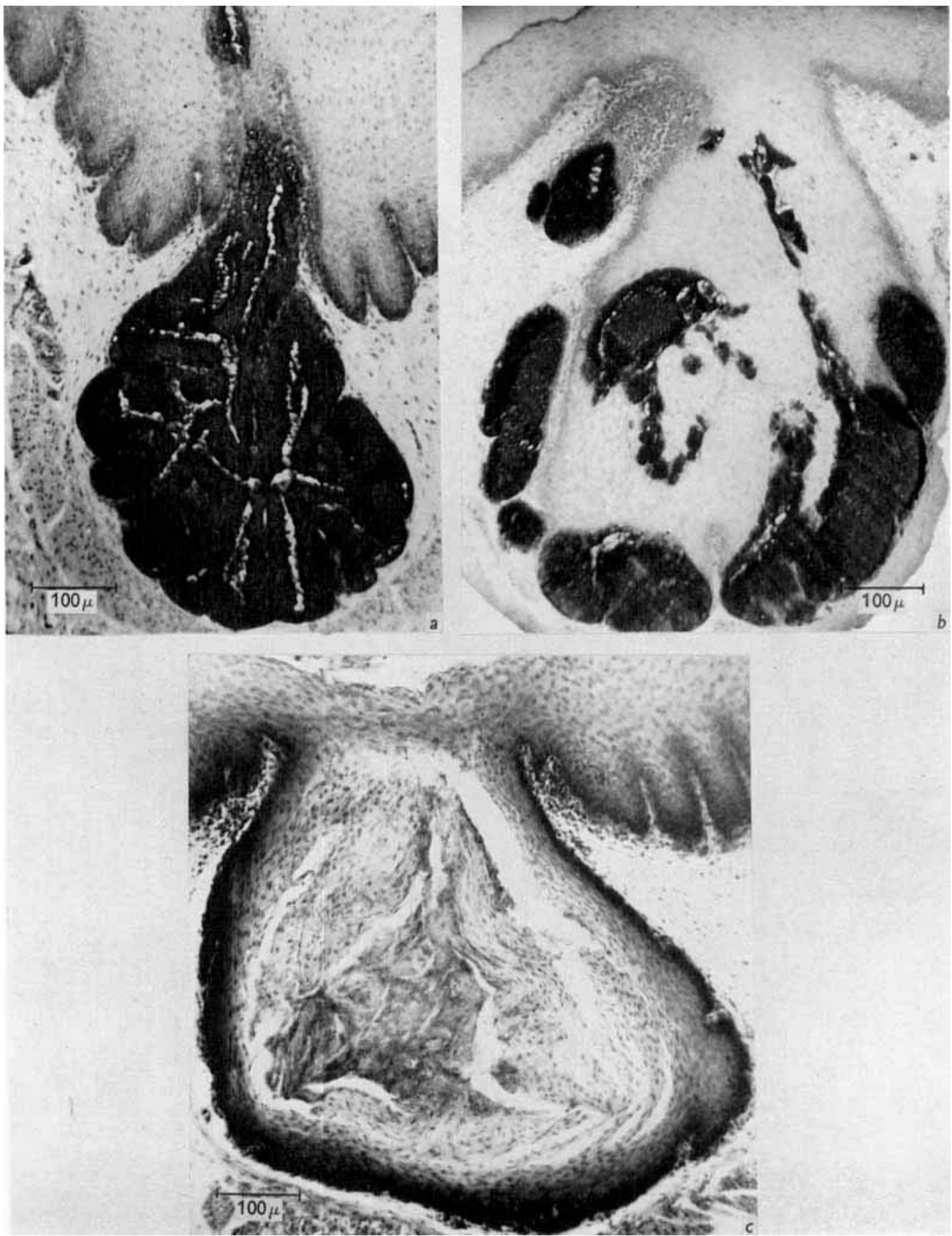

MARGARET B. AYDELOTTE 

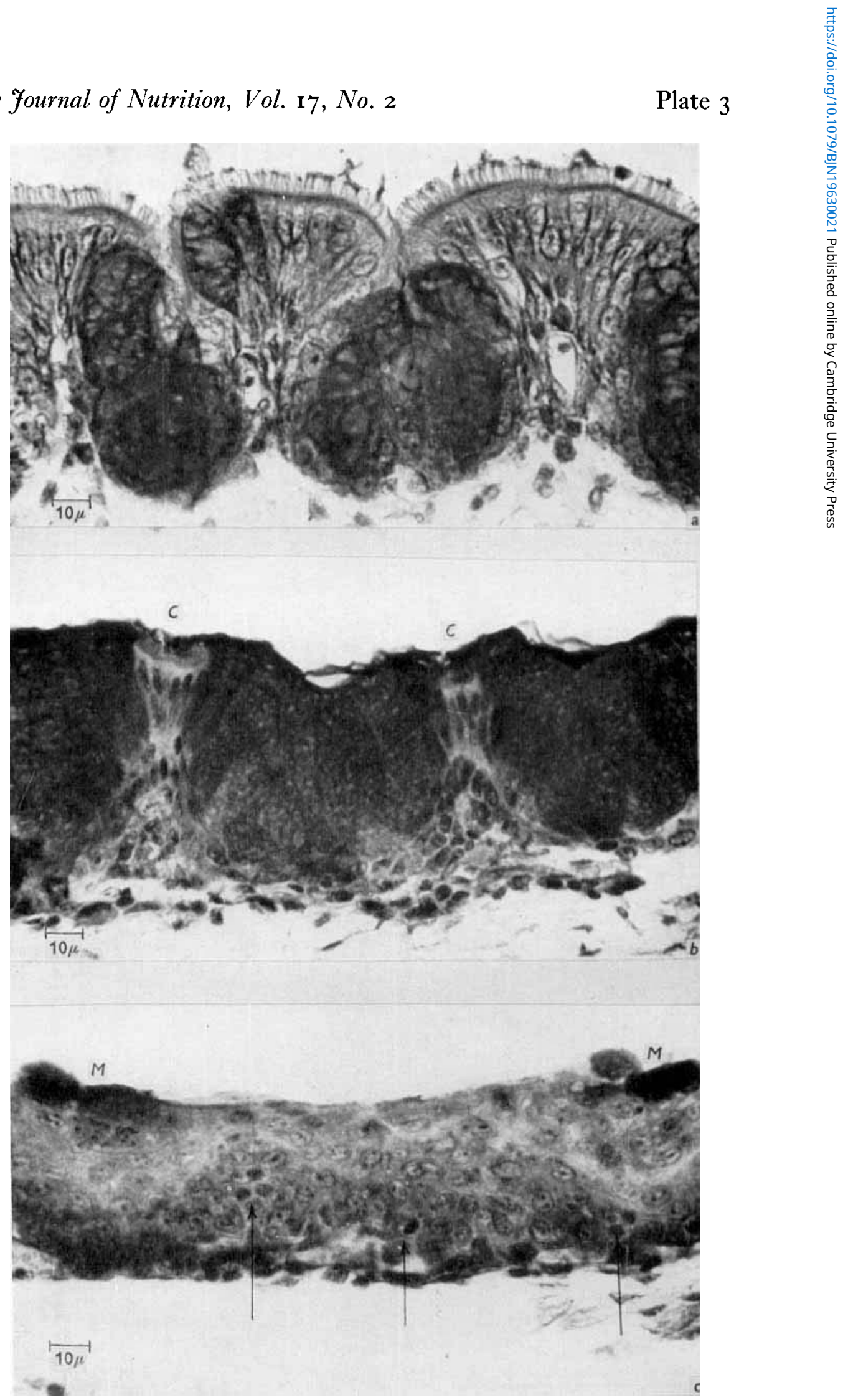